\title{
Multi-Hamiltonian structure of equations of hydrodynamic type
}

Article in Journal of Mathematical Physics · November 1990

DOI: $10.1063 / 1.529012$

CITATIONS

28

2 authors, including:

Hasan Gümral

Yeditepe University

43 PUBLICATIONS 160 CITATIONS

SEE PROFILE
READS

28

All content following this page was uploaded by Hasan Gümral on 02 June 2014. 


\title{
Multi-Hamiltonian Structure \\ of \\ Equations of Hydrodynamic Type
}

\author{
H. Gümral \\ Istanbul Technical University \\ Physics Department \\ 80626 Maslak, Istanbul, Turkey \\ and \\ Y. Nutku \\ Bilkent University \\ Department of Mathematics \\ 06533 Bilkent, Ankara, Turkey
}

J. Math. Phys. 31 (1990) 2606-2611.

\begin{abstract}
We complete the discussion of the Hamiltonian structure of 2-component equations of hydrodynamic type by presenting the Hamiltonian operators for Euler's equation governing the motion of plane sound waves of finite amplitude and another quasi-linear second order wave equation. There exists a doubly infinite family of conserved Hamiltonians for the equations of gas dynamics which degenerate into one, namely the Benney sequence, for shallow water waves. We present further infinite sequences of conserved quantities for these equations. In the case of multi-component equations of hydrodynamic type, we show that Kodama's generalization of the shallow water equations admits bi-Hamiltonian structure.
\end{abstract}




\section{Introduction}

Historically, equations of hydrodynamic type ${ }^{[1]}$ first arose as quasi-linear second order wave equations in $1+1$ dimensions. Equations of Euler ${ }^{[2]}$, Poisson ${ }^{[3]}$ and Born-Infeld $^{[4]}$ are examples of wave equations which can be written as a pair of first order equations of hydrodynamic type. The equations of gas dynamics

$$
\begin{aligned}
& u_{t}+u u_{x}+v^{\gamma-2} v_{x}=0, \\
& v_{t}+v u_{x}+u v_{x}=0,
\end{aligned}
$$

which are obtained from an Eulerian description of motion, provide the prototype of such equations. Recently Kodama ${ }^{[5]}$ has discussed the reduction of the dispersionless Kadomtsev-Petviashvili ( $\mathrm{dKP}$ ) equation which is based on the compatibility of first order equations

$$
\begin{aligned}
& u_{y}^{i}=A_{j}^{i} u_{x}^{j}, \\
& u_{t}^{i}=B_{j}^{i} u_{x}^{j}, \quad i=1,2, \ldots, n
\end{aligned}
$$

which are equations of hydrodynamic type in $2+1$ dimensions.

The Hamiltonian structure of the equations of gas dynamics $[6],[7],[8]$ which in particular include the shallow water equations ${ }^{[9],[10]}$, the Poisson ${ }^{[7]}$ and BornInfeld $^{[11]}$ equations were discussed earlier. In this paper we shall conclude the discussion of the Hamiltonian structure of these 2-component systems by presenting two further examples. First we have Euler's equation

$$
\Psi_{t t}-\left(1+\Psi_{x}\right)^{-\left(1+\gamma^{\prime}\right)} \Psi_{x x}=0
$$

governing the propagation of plane sound waves of finite amplitude and finally another quasi-linear second order wave equation

$$
\Psi_{t t}-e^{\Psi_{x}} \Psi_{x x}=0
$$

which is related to a system considered in ref.[8] . Euler's equation results from a Lagrangian, whereas eqs.(1) are obtained from an Eulerian description of motion. However, we shall show that, even though the equations of motion look quite different in different representations, they have the same Hamiltonian operators in common provided $\gamma+\gamma^{\prime}=2$.

We shall conclude by presenting the bi-Hamiltonian structure of the 3 - and 4-component generalizations of the shallow water equations which was proposed by Kodama ${ }^{[5]}$. The derivation of the second Hamiltonian operator and proof of Jacobi's identities for multi-component equations of hydrodynamic type consist of a generalization of the processes described in detail in refs. [7] and [8]. We shall assume familiarity with these papers and present only the new results unless the generalization is not entirely straight-forward. 


\section{Gas Dynamics Hierarchy}

The equations of gas dynamics were shown to admit quadri-Hamiltonian structure $^{[7],[8]}$

$$
\begin{aligned}
\mathbf{u}_{t} & =-J_{0} \mathbf{E}\left(H_{1}^{E}\right)=-J_{1} \mathbf{E}\left(H_{0}^{E}\right) \\
& =-\frac{1}{\gamma} J_{2} \mathbf{E}\left(H_{-1}^{E}\right)=-J_{3} \mathbf{E}\left(H^{V}\right) \quad \gamma \neq 0
\end{aligned}
$$

where $u^{1}=v, u^{2}=u$ are components of the vector $\mathbf{u}$ and $\mathbf{E}$ denotes the Euler operator, or the variational derivative with respect to $\mathbf{u}$. The Hamitonian operators $J_{i}, i=0,1,2,3$ are skew-adjoint matrices of differential operators satisfying the Jacobi identities. The first three of these are first order and mutually compatible, whereas the last one is third order and incompatible with the rest. The bi-Hamiltonian structure is given by

$$
\begin{gathered}
J_{0}=\sigma_{1} D \quad D=\frac{\partial}{\partial x} \\
J_{1}=\frac{1}{\gamma}\left(\begin{array}{cc}
v D+D v & (\gamma-2) D u+u D \\
D u+(\gamma-2) u D & v^{\gamma-2} D+D v^{\gamma-2}
\end{array}\right), \gamma \neq 0
\end{gathered}
$$

where $\sigma_{1}$ is the Pauli matrix. By Magri's theorem ${ }^{[12]}$, the recursion operator ${ }^{[13]}$

$$
R=J_{1} J_{0}^{-1}
$$

generates, in general, two infinite families of conserved quantities. (See section 5 for a discussion of the exceptional case $\gamma=2$.) The third Hamiltonian operator is given by

$$
J_{2}=\left(\begin{array}{cc}
u v D+D u v & {\left[\frac{1}{2}(\gamma-2) u^{2}+\frac{v^{\gamma-1}}{(\gamma-1)}\right]+} \\
D\left[\frac{1}{2} u^{2}+\frac{v^{\gamma-1}}{(\gamma-1)}\right]+ & {\left[\frac{1}{2} u^{2}+\frac{v^{\gamma-1}}{(\gamma-1)}\right] D} \\
{\left[\frac{1}{2}(\gamma-2) u^{2}+\frac{v^{\gamma-1}}{(\gamma-1)}\right] D} & u v^{\gamma-2} D+D u v^{\gamma-2}
\end{array}\right),
$$

We note that for the case of shallow water waves, $\gamma=2$, this operator is trivially related to the earlier ones by

$$
J_{2}=R^{2} J_{0}
$$

but for a generic $\gamma, J_{2}$ is a new nontrivial Hamilton Finally ${ }^{[14],[8]}$, we have the fourth Hamiltonian operator

$$
J_{3}=D U_{x}^{-1} D U_{x}^{-1} \sigma_{1} D
$$


where

$$
U=\left(\begin{array}{cc}
u & v \\
\frac{v^{\gamma-2}}{\gamma-2} & u
\end{array}\right)
$$

and this gives rise to the first order conserved density

$$
H^{V}=\frac{v_{x}}{u_{x}^{2}-v^{\gamma-3} v_{x}^{2}}
$$

which is due to Verosky ${ }^{[15]}$.

Equations of polytropic gas dynamics admit the following two sequences of conserved densities

$$
\begin{aligned}
H_{-1}^{E} & =\gamma v, \\
H_{0}^{E} & =u v, \\
H_{1}^{E}= & \frac{1}{2} u^{2} v+\frac{v^{\gamma}}{\gamma(\gamma-1)}, \quad \gamma \neq 0,1 \\
H_{2}^{E}= & \frac{1}{6} u^{3} v+\frac{u v^{\gamma}}{\gamma(\gamma-1)}, \\
H_{3}^{E}= & \frac{1}{24} u^{4} v+\frac{u^{2} v^{\gamma}}{2 \gamma(\gamma-1)}+\frac{v^{2 \gamma-1}}{2 \gamma(\gamma-1)^{2}(2 \gamma-1)}, \\
H_{0}^{L}= & u, \\
H_{1}^{L}= & \frac{1}{2}(\gamma-2) u^{2}+\frac{v^{\gamma-1}}{(\gamma-1)}, \\
H_{2}^{L}= & \frac{1}{6}(\gamma-2) u^{3}+\frac{u v^{\gamma-1}}{(\gamma-1)}, \\
H_{3}^{L}= & \frac{1}{24}(\gamma-2) u^{4}+\frac{1}{2} \frac{u^{2} v^{\gamma-1}}{2(\gamma-1)}+\frac{v^{2(\gamma-1)}}{2(\gamma-1)^{2}(2 \gamma-3)},
\end{aligned}
$$

with superscripts $E$ and $L$ denoting the Euler and Lagrange hierarchies, respectively. The recursion operator keeps the two hierarchies separate.

It is the third conserved quantity in the Euler hierarchy $H_{1}^{E}$ which acts as the Hamiltonian function for various physically interesting quasi-linear wave equations. The exceptional case $\gamma=1$ where

$$
H_{1}^{E}=\frac{1}{2} u^{2} v+v(\ln v-1)
$$

corresponds to Poisson's equation in nonlinear acoustics, while $\gamma=-1$, which is also known as Chaplygin gas, yields the Born-Infeld equation and classical shallow water equations are obtained for $\gamma=2$. The first nontrivial conserved quantity in the Lagrange hierarchy $H_{1}^{L}$ has not played a role in the equations which were discussed earlier. 


\section{Euler's Equation}

In 1757 Euler considered finite amplitude plane sound waves and obtained the quasi-linear second order partial differential equation( 3$)$ where $\Psi(x, t)$ is the displacement in a Lagrangian description of motion. This equation has been the subject of extensive classic investigations ${ }^{[16]}$. We shall now show that the second order equation which results from the use of the Hamiltonian function $H_{1}^{L}$ in the Lagrange hierarchy is precisely Euler's equation.

Equation (3) is a member of a class of completely integrable nonlinear wave equations which admits infinitely many conservation laws ${ }^{[18]}$. Thus, through the introduction of a new potential $\Phi$, Euler's equation can be realized as the integrability condition of a first order system according to the formalism of refs.[7],[18]. Such a system is given by

$$
\begin{aligned}
& \Phi_{t}=-\gamma^{\prime}-1\left(1+\Psi_{x}\right)^{-\gamma^{\prime}}, \gamma^{\prime} \neq 0 \\
& \Psi_{t}=\Phi_{x}
\end{aligned}
$$

and using the velocity fields

$$
\begin{aligned}
& u=\Phi_{x}, \\
& v=1+\Psi_{x},
\end{aligned}
$$

we find the evolution equations

$$
\begin{aligned}
& u_{t}=v^{-\left(1+\gamma^{\prime}\right)} v_{x}, \\
& v_{t}=u_{x},
\end{aligned}
$$

which belong to the class of equations of hydrodynamic type. Equations( 19) admit primary Hamiltonian structure

$$
\mathbf{u}_{t}=-\frac{1}{\gamma^{\prime}} J_{0} \mathbf{E}\left(H_{1}^{L}\right)
$$

with Hamiltonian density

$$
H_{1}^{L}=-\frac{1}{2} \gamma^{\prime} u^{2}+\frac{v^{1-\gamma^{\prime}}}{1-\gamma^{\prime}} \quad \gamma^{\prime} \neq 0,1
$$

and comparison with eq.( 15) leads to the identification

$$
\gamma+\gamma^{\prime}=2 .
$$

The choice of the velocity fields in eqs.( 18) and the identification( 22) were designed to cast Euler's equation into a form whereby its bi-Hamiltonian structure is manifest. Thus

$$
\mathbf{u}_{t}=-\frac{1}{\gamma^{\prime}} J_{0} \mathbf{E}\left(H_{1}{ }^{L}\right)=\frac{\gamma^{\prime}}{\gamma^{\prime}-2} J_{1} \mathbf{E}\left(H_{0}{ }^{L}\right)
$$


where the Hamiltonian operators are identical to those given in eq.( 6) and eq.( 7 ). It is not possible to extend the recursion relation beyond this because $H_{-1}^{L}$ is not defined. On the other hand using the recursion operator we can construct the next completely integrable equation

$$
\Psi_{t t}+\frac{1}{\gamma^{\prime}\left(1+\Psi_{x}\right)^{\gamma^{\prime}}} \Psi_{x t}+\left[\frac{1-3 \gamma^{\prime}}{\gamma^{\prime 2}\left(1-\gamma^{\prime}\right)} \frac{1}{\left(1+\Psi_{x}\right)^{2 \gamma^{\prime}}}-\frac{2 \Psi_{t}}{\left(1+\Psi_{x}\right)^{1+\gamma^{\prime}}}\right] \Psi_{x x}=0
$$

for which the first Hamiltonian function is ${H_{2}}_{2}{ }^{2}$ and we can therefore extend the recursion relation to include $J_{2}$. The third order Hamiltonian operator $J_{3}$ exists for all of these equations. Quasi-linear wave equations in the hierarchy of Euler's equation admit quadri-Hamiltonian structure. The first three Hamiltonian structures of Euler's equation were obtained by Kupershmidt ${ }^{[17]}$ using a different approach.

\section{The equation $\Psi_{t t}=e^{\Psi_{x}} \Psi_{x x}$}

In our discussion of the multi-Hamiltonian structure of Euler's equation we have introduced the first order system (17) which is not defined for $\gamma^{\prime}=0$. On the other hand the resulting equations of hydrodynamic type (19) are defined for all $\gamma^{\prime}$ but they are not bi-Hamiltonian in the exceptional case $\gamma^{\prime}=0$. This gap is filled by the equation (4) which is obtained by an interchange of the roles of $x$ and $t$ in Euler's equation for $\gamma^{\prime}=0$.

We consider the first order system

$$
\begin{aligned}
& \Phi_{t}=e^{\Psi_{x}}-1 \\
& \Psi_{t}=\Phi_{x}
\end{aligned}
$$

the integrability conditions of which result in eq.( 4) for the potential $\Psi$, while $\Phi$ satisfies

$$
\Phi_{x x}-\left(1+\Phi_{t}\right)^{-1} \Phi_{t t}=0,
$$

Euler's equation for $\gamma^{\prime}=0$ with the roles of $x$ and $t$ interchanged. Defining the velocity fields $u=\Phi_{x}$ and $v=\Psi_{x}$ we obtain the hydrodynamic system

$$
\begin{aligned}
& u_{t}=e^{v} v_{x}, \\
& v_{t}=u_{x}
\end{aligned}
$$

which are quadri-Hamiltonian equations of hydrodynamic type. The bi-Hamiltonian structure is defined by the operators $\hat{J}_{0}=J_{0}$ and

$$
\hat{J}_{1}=\left(\begin{array}{cc}
e^{v} D+D e^{v} & u D \\
D u & 2 D
\end{array}\right) .
$$


The third Hamiltonian operator

$$
\hat{J}_{2}=\left(\begin{array}{cc}
u e^{v} D+D u e^{v} & \left(\frac{1}{2} u^{2}+e^{v}\right) D+D e^{v} \\
e^{v} D+D\left(e^{v}+\frac{1}{2} u^{2}\right) & u D+D u
\end{array}\right)
$$

which is trivially related to the first two was obtained earlier ${ }^{[8]}$. These operators are compatible. There is also a third order Hamiltonian operator for eqs.( 27)

$$
\begin{gathered}
\hat{J}_{3}=D \hat{U}_{x}^{-1} D \hat{U}_{x}^{-1} \sigma_{1} D, \\
\hat{U}=\left(\begin{array}{cc}
u & v \\
e^{v} & u
\end{array}\right)
\end{gathered}
$$

which is incompatible with the rest. These equations admit the first order conserved density

$$
\hat{H}=\frac{v_{x}}{u_{x}^{2}-e^{v} v_{x}^{2}}
$$

which is analoguous to Verosky's result.

The recursion operator $\hat{R}=\hat{J}_{1} J_{0}^{-1}$ generates infinitely many conserved quantities the first few of which are

$$
\begin{aligned}
\hat{H}_{-1}^{E} & =v \\
\hat{H}_{0}^{L} & =u \\
\hat{H}_{1}^{L} & =\frac{1}{2} u^{2}+e^{v} \\
\hat{H}_{2}^{L} & =\frac{1}{3} u^{3}+2 u e^{v} \\
\hat{H}_{3}^{L} & =\frac{1}{4} u^{4}+3 u^{2} e^{v}+\frac{3}{2} e^{2 v}, \hat{H}_{4}^{L} \\
\frac{1}{5} u^{5}+4 u^{3} e^{v}+6 u e^{2 v} &
\end{aligned}
$$

Apart from the first one all the conserved quantities in this sequence are related to those in the Lagrangian sequence of generalized gas dynamics. The recursion operator $\hat{R}$ when applied to the conserved density $\hat{H}_{-1}^{E}$ in the Eulerian sequence generates the analogue of the Lagrangian sequence. This situation is in agreement with the fact that the two sequences of conserved quantities for gas dynamics become identical ${ }^{[7]}$ to the Benney sequence ${ }^{[19]}$ when $\gamma=2$, which corresponds to $\gamma^{\prime}=0$ for Euler's equation. 


\section{Further Conserved Quantities for Shallow Wa- ter Equations}

Two-component equations of hydrodynamic type admit conserved quantities which satisfy a linear second order pde in 2 variables $^{[18]}$. From general theory we know that the solution of such an equation contains two arbitrary functions. Indeed, for a generic $\gamma$ the two infinite families of conserved Hamiltonians of the Eulerian and the Lagrangian sequences form a complete set in terms of which we can express these arbitrary functions. However, it was already noted in ref.[7] that for the case of shallow water waves $(\gamma=2)$ these two sequences are no longer linearly independent and we have just seen above that the same phenomenon occurs for $\gamma^{\prime}=0$. This degeneration of the Eulerian and the Lagrangian sequences into one, namely the Benney sequence, results in the loss of an arbitrary function. Thus for $\gamma=2$, or $\gamma^{\prime}=0$ we are missing an infinite set of conserved quantities.

Possible end/starting elements of such a missing sequence of conserved quantities are the Casimirs $C$ which satisfy

$$
J_{1} \mathbf{E}(C)=0
$$

and for shallow water equations we find that these distinguished functions are given by

$$
C=\sqrt{u^{2}-4 v}
$$

and $u$ itself. For the non-trivial Casimir 35 simple waves satisfying

$$
u^{2}=4 v
$$

form a dividing line in the discussion which follows and appropriate restrictions must be imposed in order to insure that the arguments of the square roots are

positive. The Benney sequence and the Casimirs can be obtained in various limits of Manin

$$
M(k)=\sqrt{(u+k)^{2}-4 v}-(u+k)
$$

for conserved quantities satisfied by the shallow water equations. We have the following infinite sequences of conserved quantities which include the non-trivial Casimir

$$
\ldots \rightarrow \frac{u}{\sqrt{u^{2}-4 v}} \rightarrow \sqrt{u^{2}-4 v} \rightarrow 0, \quad u^{2}-4 v>0
$$

which was pointed out in ref. [21] and

$$
0 \rightarrow \sqrt{4 v-u^{2}} \rightarrow 4 v \sin ^{-1}\left(\frac{u}{2 \sqrt{v}}\right)+u \sqrt{4 v-u^{2}}+\ldots, u^{2}-4 v<0,
$$

where arrow indicates the sense of the recursion operator. Once again ${ }^{[8]} \partial / \partial u$ acts as the inverse of the recursion operator. 


\section{Kodama's Generalization of Shallow Water Equa- tions}

Kodama $^{[5]}$ has shown that the reduction of the dKP equation which results in the shallow water equations leads to the following generalization for a 3 component field

$$
\left(\begin{array}{c}
v \\
w \\
u
\end{array}\right)_{t}=\left(\begin{array}{ccc}
u & w & v \\
0 & u & w \\
1 & 0 & u
\end{array}\right)\left(\begin{array}{c}
v \\
w \\
u
\end{array}\right)_{x}
$$

which are equations of hydrodynamic type. They admit an infinite sequence of conserved Hamiltonians

$$
\begin{aligned}
H_{-1}= & 2 v, \\
H_{0}= & u v+\frac{1}{2} w^{2}, \\
H_{1}= & \frac{1}{2}\left(u^{2} v+v^{2}+u w^{2}\right), \\
H_{2}= & \frac{1}{4} u^{3} v+\frac{3}{4} u v^{2}+\frac{3}{8} u^{2} w^{2}+\frac{3}{8} v w^{2}, \\
& \cdots
\end{aligned}
$$

analoguous to the Benney sequence for shallow water waves. Kodama has further noted that his equations can be written in Hamiltonian form

$$
\mathbf{u}_{t}=J_{0} \mathbf{E}\left(H_{1}\right)
$$

where

$$
J_{0}=\left(\begin{array}{ccc}
0 & 0 & D \\
0 & D & 0 \\
D & 0 & 0
\end{array}\right)
$$

and $\mathbf{u}=(v, w, u)$ in that order. We shall now show that Kodama's equations admit bi-Hamiltonian structure.

We shall seek a second Hamiltonian operator for eqs.( 40) using a procedure similar to that presented in ref.[7] for two component equations. Since the calculations are straight-forward and lengthy we shall only present the results. The second Hamiltonian operator for Kodama's equations is given by

$$
J_{1}=\frac{1}{2}\left(\begin{array}{ccc}
v D+D v & w D+D \frac{w}{2} & u D \\
\frac{w}{2} D+D w & \frac{1}{4}\left(2 u-\frac{v^{2}}{w^{2}}\right) D+D \frac{1}{4}\left(2 u-\frac{v^{2}}{w^{2}}\right) & \frac{v}{2 w} D \\
D u & D \frac{v}{2 w} & \frac{3}{2} D
\end{array}\right)
$$

and it can be verified that this expression for $J_{1}$ satisfies the Jacobi identities and is compatible with $J_{0}$. The recursion operator for this bi-Hamiltonian system 
generates the infinite sequence of conserved Hamiltonians obtained by Kodama starting with $v$. But in addition there is an infinite sequence of conserved quantities starting with $w$

$$
\begin{aligned}
H_{0}^{\prime}= & w \\
H_{1}^{\prime}= & \frac{1}{2} u w+\frac{1}{4} \frac{v^{2}}{w}, \\
H_{2}^{\prime}= & \frac{3}{8} u^{2} w+\frac{3}{4} v w+\frac{3}{8} \frac{u v^{2}}{w}-\frac{1}{32} \frac{v^{4}}{w^{3}}, \\
H_{3}^{\prime}= & \frac{5}{16} u^{3} w+\frac{15}{8} u v w+\frac{5}{16} w^{3}+\frac{15}{32} \frac{u^{2} v^{2}}{w}-\frac{5}{64} \frac{u v^{4}}{w^{3}} \\
& \quad+\frac{5}{16} \frac{v^{3}}{w}+\frac{1}{128} \frac{v^{6}}{w^{5}},
\end{aligned}
$$

Thus we have 2 infinite families of conserved Hamiltonians for the 3-component Kodama equations which are given by rational functions. However we are missing an infinite family conserved quantities.

As in our earlier discussion of the conservation laws for the classical shallow water equations, the missing infinite sequence of conserved quantities must include the Casimir. For the Hamiltonian operator(44) we find that

$$
C=\left(3 v-u^{2}\right)^{1 / 2}\left(\frac{(1+\xi)^{1 / 2}-1}{(1+\xi)^{1 / 2}+1}\right)^{1 / 6}, \quad \xi=\frac{16}{(27)^{2}} \frac{\left(3 v-u^{2}\right)^{3}}{\left(w^{2}+\frac{4}{27} u^{3}-\frac{2}{3} u v\right)^{2}}
$$

is the non-trivial Casimir. Once again $u$ is also a Casimir.

Finally, we note that the 4-component form of Kodama's equation

$$
\left(\begin{array}{l}
v \\
r \\
s \\
u
\end{array}\right)_{t}=\left(\begin{array}{llll}
u & s & r & v \\
0 & u & s & r \\
0 & 0 & u & s \\
1 & 0 & 0 & u
\end{array}\right)\left(\begin{array}{c}
v \\
r \\
s \\
u
\end{array}\right)_{x}
$$

is also a bi-Hamiltonian system with

$$
J_{0}=\left(\begin{array}{cccc}
0 & 0 & 0 & D \\
0 & 0 & D & 0 \\
0 & D & 0 & 0 \\
D & 0 & 0 & 0
\end{array}\right)
$$

and

$$
J_{1}=\frac{1}{6}\left(\begin{array}{cccc}
6 v D+3 v_{x} & 5 r D+2 r_{x} & 4 s D+s_{x} & 3 u D \\
5 r D+3 r_{x} & 4 m D+2 m_{x} & 3 n D+n_{x} & 2 p D \\
4 s D+3 s_{x} & 3 n D+2 n_{x} & 2 q D+q_{x} & (r / s) D \\
3 D u & 2 D p & D(r / s) & 4 D
\end{array}\right)
$$


where

$$
\begin{aligned}
m & =s-\frac{1}{2} \frac{v^{2}}{s^{2}}+\frac{v r^{2}}{s^{3}}-\frac{1}{2} \frac{r^{4}}{s^{4}}, \\
p & =\frac{v}{s}-\frac{1}{2} \frac{r^{2}}{s^{2}} \\
n & =u-\frac{v r}{s^{2}}+\frac{2}{3} \frac{r^{3}}{s^{3}}, \\
q & =\frac{v}{s}-\frac{r^{2}}{s^{2}} .
\end{aligned}
$$

The n-component Kodama equations are evidently also bi-Hamiltonian but the explicit expression for the second Hamiltonian operator is rather involved.

We note that in Kodama's equations (40) the limit $w \rightarrow 0$ is well-defined and yields the classical shallow water equations (1) with $\gamma=2$. However, we have found that this limit is not defined for the second Hamiltonian operator (44) of Kodama's equations. Thus it is not possible to obtain the Hamiltonian operator of eq.(7) starting from eq.(44). Similar remarks apply to the 4-c (47) as well. This may seem surprising at first sight, indeed it was the source of failure of early easy guesses for the possible bi-Hamiltonian structure of eqs.(40), but the non-existence of the $w \rightarrow 0$ limit in eq.(44) can be traced back to the following: There is a dimensional reason for the appearance of inverse powers of $w$ in the second Hamiltonian operator $J_{1}$ for Kodama's equations. In the n-component Kodama equations the variables $\left\{u^{i}\right\}, i=1,2, . ., n$ carry the dimension

$$
\left[u^{i}\right]=\frac{2 n-1-i}{n-1}
$$

and this results in the requirement that the $i, k$ entry of the Hamiltonian operator must have the dimension

$$
\left[J^{i k}\right]=\frac{2 n-i-k}{n-1}
$$

So we must start with an Ansatz for the entries of $J^{i k}$ with the appropriate dimension (52) but if we were to exclude terms containg the inverse powers of some of the variables in this Ansatz, it can be verified that the Jacobi identities cannot be satisfied. The results presented in eqs. $(44,49)$ for the second Hamiltonian operators of Kodama's equations $(40,47)$ satisfy these dimensional considerations and the Jacobi identities.

\section{Conclusion}

We have shown that Euler's equation governing the propagation of plane sound waves of finite amplitude can be cast into form of 2-component equations of hydrodynamic type. The first Hamiltonian function of this system can be identified 
with the first conserved quantity in the Lagrangian sequence of gas dynamics. The quadri-Hamiltonian structure of gas dynamics can therefore be carried over to Euler's equation. We have further shown that the quasi-linear second order wave equation( 4) also admits quadri-Hamiltonian structure.

The multi-Hamiltonian structure of equations of hydrodynamic type is a remarkably rich subject as the results reported in refs.[7],[8] together with this paper will indicate. But so far we have mostly considered 2-component equations and only begun to investigate the multi-component case by exhibiting the bi-Hamiltonian structure of Kodama's equations generalizing the shallow water equations. The Hamiltonian structure of multi-component equations of hydrodynamic type which are obtained from different reductions of the dKP equation requires further investigation.

\section{References}

[1] B.A. Dubrovin and S.A. Novikov, Sov. Math. Dokl. 27 (1983) 665

[2] L. Euler, Hist. Acad. Berlin 11, (1757) 449

[3] S.D. Poisson, J. Ec. Polytech. ㄱ, (1808) 319

[4] M. Born and L. Infeld, Proc. Roy. Soc. (London) A144 (1934), 425

[5] Y. Kodama, Prog. Theor. Phys. Suppl. No.94 (1988) 184

[6] Y. Nutku, J. Phys.A: Math. Gen. 16, (1983) 4195

[7] Y. Nutku, J. Math. Phys. $\underline{28}$ (1987) 2579

[8] P.J. Olver and Y. Nutku, J. Math. Phys. $\underline{29}$ (1988) 1610

[9] J. Cavalcante and H.P. McKean, Physica (Utrecht) D4 (1982) 253

[10] B.A. Kupershmidt, Commun. Math. Phys.,99 (1985) 51

[11] M. Arik, F. Neyzi, Y. Nutku, P.J. Olver and J. Verosky, J. Math. Phys., $\underline{30}$ (1988) 1338

[12] F. Magri, J. Math. Phys.,19 (1978) 1356

[13] P.J.Olver," Applications of Lie Groups to Differential Equations", Graduate Texts in Mathematics (Springer, New York,1986) Vol. 107

[14] M. B. Sheftel, Func. Anal. Appl.,20 (1986) 227

[15] J. M. Verosky, J. Math. Phys., $\underline{25}$ (1984) 884

[16] Rayleigh, Proc. Roy. Soc. London, Ser A 84 (1910) 247 
[17] B.A. Kupershmidt, "Discrete Lax Equation and Differential-Difference Calculus" Societe Mathematique de France (1985)

[18] Y. Nutku, J. Math. Phys.,26 (1984) 1237

[19] D. J. Benney, Stud. Appl. Math. $\underline{52}$ (1973) 45

[20] Yu. Manin, Itogi Nauki i Tekhniki, Sov. Prob. Mat. 11 (1978) 5

[21] M. Antonowicz and A. P. Fordy, Physica D $\underline{28}$ (1987) 345 